\title{
DWF calculation of the leading order hadronic vacuum polarisation to $\mathrm{g}-2$ of the muon.
}

\author{
Eoin Kerrane* \\ School of Physics and Astronomy, University of Edinburgh \\ E-mail: eoin.kerraneded.ac.uk
}

\section{Peter Boyle}

School of Physics and Astronomy, University of Edinburgh

E-mail: paboyleeph.ed.ac.uk

\section{Luigi Del Debbio}

School of Physics and Astronomy, University of Edinburgh

E-mail: luigi.del.debbioded.ac.uk

\section{James Zanotti}

School of Physics and Astronomy, University of Edinburgh ${ }^{\dagger}$

E-mail: jzanotti@ph.ed.ac.uk

\begin{abstract}
We present a calculation [1] of the leading order hadronic contribution to the anomalous magnetic moment of the muon for a dynamical simulation of $2+1$ flavour QCD using domain wall fermions. The electromagnetic 2-point function is evaluated on the lattice gauge configurations and this is fitted to a continuous form motivated by models of vector dominance. We find broad agreement with previous lattice results for this quantity, while improvements in simulation and theory are clearly needed in order to produce satisfactorily precise results.
\end{abstract}

XXIX International Symposium on Lattice Field Theory

July 10 - 162011

Squaw Valley, Lake Tahoe, California

\footnotetext{
* Speaker.

†Present address: CSSM, The School of Chemistry and Physics, The University of Adelaide, South Australia 5005, Australia.
} 


\section{Introduction}

The anomalous magnetic moment $a$ of a lepton is a measure of the quantum contributions to the lepton-photon interaction. The result for the muon, $a_{\mu}$ comprises significant contributions from most sectors of the Standard Model and as such the effort to produce a precise computation of the theoretical prediction for $a_{\mu}$ has proven a significant challenge. This fact, along with the success of experiments in determining the physical value of $a_{\mu}$ to extraordinary precision makes $a_{\mu}$ a very important quantity. Further, the current discrepancy between the experimental and theoretical Standard Model values is playing a significant role in shaping the debate over candidate models for BSM physics.

The current uncertainty in $a_{\mu}$ is strongly dominated by hadronic contributions. This work involves the leading order hadronic contribution, which we denote as $a_{\mu}^{(2) h a d}$, the best estimate of which is currently obtained by relating the hadronic vacuum polarisation of the photon to the cross section for $e^{+} e^{-}$decay into hadrons, allowing a dispersive integral over experimental data for the cross section [2]. Despite the apparent accuracy of the results obtained from this procedure, there remain discrepancies between results from different data sets. It would be preferable to obtain the hadronic contribution to $a_{\mu}$ from a first principles approach. For this the only valid candidate is lattice QCD which alone is capable of producing quantitative results from fully non-perturbative QCD.

This quantity was first tackled through lattice computation in quenched simulations first with domain wall fermions [3] , followed by a calculation with improved Wilson fermions [4]. The first dynamical simulation followed [5, 6] using 2+1 flavour staggered quarks, and several studies of this quantity are ongoing, using 2 flavours of improved Wilson fermions [7] and twisted mass fermions [8]. We present a calculation of $a_{\mu}^{(2) h a d}$ from a dynamical simulation of $2+1$ flavour QCD with domain wall fermions.

As described in [3] the expression for the hadronic vacuum polarisation contribution to $a m_{\mu}$ can be expressed

$$
a_{\mu}^{(2) h a d}=\left(\frac{\alpha}{\pi}\right)^{2} \int_{0}^{\infty} d Q^{2} f\left(Q^{2}\right) \times \hat{\Pi}\left(Q^{2}\right)
$$

where $\hat{\Pi}\left(Q^{2}\right)$ is the infra-red subtracted transverse part of the hadronic vacuum polarisation

$$
\hat{\Pi}\left(Q^{2}\right)=\Pi\left(Q^{2}\right)-\Pi(0) \quad \Pi_{\mu v}(q)=\left(q^{2} g_{\mu v}-q_{\mu} q_{v}\right) \Pi\left(q^{2}\right)
$$

The hadronic vacuum polarisation function $\Pi_{\mu v}(q)$ can be computed as the Fourier-transformed two-point correlator

$$
\Pi_{\mu v}(q)=\int d^{4} x e^{i q \cdot(x-y)}\left\langle J_{\mu}(x) J_{v}(y)\right\rangle
$$

involving the electromagnetic current $J_{\mu}(x)=\sum_{i} Q_{i} \bar{\psi}^{i} \gamma_{\mu} \psi^{i}$ where $\psi^{i}$ is the quark field of flavour $i$ and $Q^{i}$ is its charge. The path-integral used in the expectation value in (1.3) will involve only hadronic fields, i.e. quarks and gluons.

\section{Simulation}

Our computation is performed using configurations generated by the RBC \& UKQCD collaborations as part of their program of investigation using $2+1$ flavours of domain-wall fermions. We 
investigate three lattice volumes, each with several ensembles at different values of the light quark mass $m_{u}$. The parameters of these ensembles are given in Table 1 . The ensembles at $\beta=1.75$ have been generated using a dislocation suppressing determinant ratio (DSDR) in conjunction with the Iwasaki gauge action, with a fifth dimension whose extent is $L_{5}=32[9,10]$. The lighter of these ensembles is very near to the physical point with a pion mass of $m_{\pi} \simeq 180 \mathrm{MeV}$. The other ensembles used only the Iwasaki action and $L_{5}=16[11,12]$.

\begin{tabular}{c|c|c|c|c|c}
$V$ & $\beta$ & $a^{-1} \mathrm{GeV}$ & $\hat{q}_{\min }^{2} \mathrm{GeV}^{2}$ & $a m_{h}$ & $a m_{u}$ \\
\hline \hline $24^{3} \times 64$ & 2.13 & $1.73(2)$ & 0.028 & 0.04 & 0.02 \\
$24^{3} \times 64$ & 2.13 & $1.73(2)$ & 0.028 & 0.04 & 0.01 \\
$24^{3} \times 64$ & 2.13 & $1.73(2)$ & 0.028 & 0.04 & 0.005 \\
$32^{3} \times 64$ & 2.25 & $2.28(3)$ & 0.05 & 0.03 & 0.008 \\
$32^{3} \times 64$ & 2.25 & $2.28(3)$ & 0.05 & 0.03 & 0.006 \\
$32^{3} \times 64$ & 2.25 & $2.28(3)$ & 0.05 & 0.03 & 0.004 \\
$32^{3} \times 64$ & 1.75 & $1.375(9)$ & 0.018 & 0.045 & 0.0042 \\
$32^{3} \times 64$ & 1.75 & $1.375(9)$ & 0.018 & 0.045 & 0.001
\end{tabular}

Table 1: Parameters of the lattice ensembles used in our study.

\subsection{Vacuum polarisation}

We compute the lattice vacuum polarisation as

$$
\widetilde{\Pi}_{\mu v}(\hat{q}) \equiv Z_{V} \sum_{i} Q_{i}^{2} \sum_{x} e^{i q x} a^{6}\left\langle\mathscr{V}_{\mu}^{i}(x) V_{v}^{i}(0)\right\rangle .
$$

where we have omitted the flavour-nondiagonal terms as they contain only "disconnected" contributions which are expected to be sub-dominant, as will be discussed further below.

At the sink we use the DWF conserved vector current [13]

$$
\mathscr{V}_{\mu}^{i}(x)=\sum_{s=1}^{L_{5}} \frac{1}{2}\left[\bar{\psi}^{i}(x+\hat{\mu}, s)\left(1+\gamma_{\mu}\right) U_{\mu}^{\dagger}(x) \psi^{i}(x, s)-\bar{\psi}^{i}(x, s)\left(1-\gamma_{\mu}\right) U_{\mu}(x) \psi^{i}(x+\hat{\mu}, s)\right]
$$

while at the source we have the local vector current $V_{v}^{i}(x)=\bar{q}^{i}(x) \gamma_{v} q^{i}(x)$ where $q^{i}(x)=P_{+} \psi^{i}\left(x, L_{5}-\right.$ $1)+P_{-} \psi^{i}(x, 0)$, and $P_{ \pm}=\frac{1}{2}\left(1 \pm \gamma_{5}\right)$. Because of the use of the local vector current, a factor of the vector current renormalisation constant, $Z_{V}$, is included in our definition of the vacuum polarisation.

Of the two Wick-contractions arising from this correlator, we compute only the connected one. We leave the evaluation of the disconnected contribution for future work, but note that it is expected to be suppressed relative to the connected contribution [14]. This argument is also the motivation for neglecting the flavour-nondiagonal terms, and we will make an estimate of the systematic uncertainty that results in our conclusions.

\section{Deducing $a_{\mu}^{(2) h a d}$}

In order to infer the value of $a_{\mu}^{(2) h a d}$ from our data we must carry out the integral (1.1) which we split into high and low momentum regions at some momentum cut $Q_{C}^{2}$ 


$$
a_{\mu}^{(2) h a d}=4 \alpha^{2}\left[\int_{0}^{Q_{C}^{2}} d Q^{2} f\left(Q^{2}\right) \times \hat{\Pi}\left(Q^{2}\right)+\int_{Q_{C}^{2}}^{\infty} d Q^{2} f\left(Q^{2}\right) \times \hat{\Pi}\left(Q^{2}\right)\right] .
$$

A continuous description of $\Pi\left(Q^{2}\right)$ at low momenta is obtained by performing a fit to our lattice data, which allows us to perform the low $Q^{2}$ integral. The value of $\Pi(0)$ from this fit combined with a high-momentum description of $\Pi\left(Q^{2}\right)$ from perturbation theory allows us to perform the high momentum integral. As we shall see, the integral is strongly dominated by the low momentum contribution.

\subsection{Fitting the low $Q^{2}$ region}

We have attempted to fit a continuous form to our lattice data for the vacuum polarisation using a number of different fit forms. The effect that the choice of fit function can have on the result for $a_{\mu}^{(2) h a d}$ has been highlighted in previous studies [6], and this behoves us to ensure that the systematics with regard to this choice are under control. Ref. [6] also illustrated the use of a fit form originating in the expression for the vacuum polarisation calculated in chiral perturbation theory. The dominant component of this expression is due to the vector meson contribution.

Motivated by this expression the fit-form we use is closely related, differing only in the inclusion of the contribution of an additional vector resonance,

$$
\Pi\left(Q^{2}\right)=A-\frac{F_{1}^{2}}{Q^{2}+m_{1}^{2}}-\frac{F_{2}^{2}}{Q^{2}+m_{2}^{2}} .
$$

We fit the lattice vacuum-polarisation data in two ways: firstly using $A, F_{1,2}$ and $m_{1,2}$ as free parameters, and also by fixing the parameter $m_{1}$ to the mass of the vector meson $m_{\mathrm{V}}$ as measured in [12]. This we do by constraining $m_{1}$ to lie in the one-sigma band defined by the estimate of $m_{\mathrm{V}}$ and its variance.

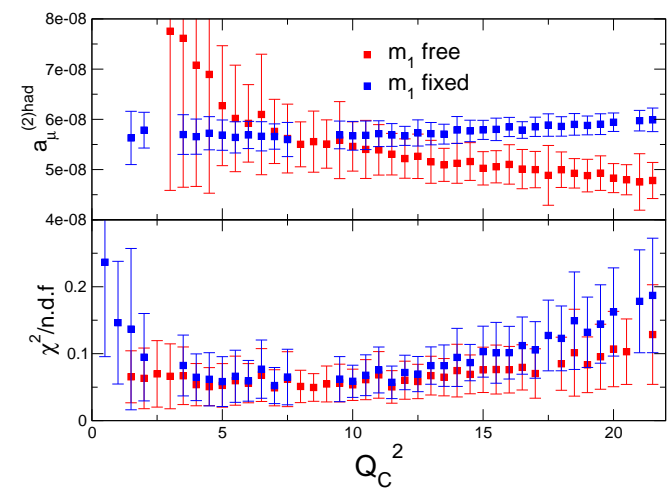

(a) Properties of fits to the lattice vacuum polarisation.

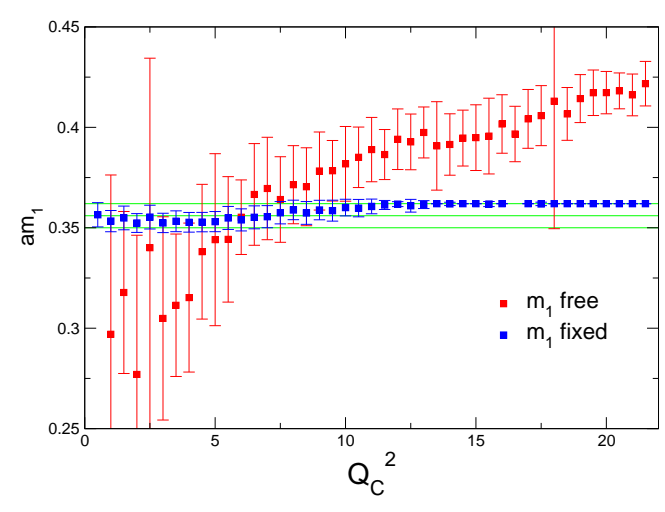

(b) Value of the fit parameter $a m_{1}$.

Figure 1: Properties of fits to the lattice vacuum polarisation using the ansatz (3.2) on the $\beta=2.25$ lattice at $a m_{u}=0.004$

The behaviour of such fits is shown in Fig. 1a. Clearly such a form is a very good representation of the data, over practically the whole range of $Q_{C}^{2}$. In addition the results for $a_{\mu}^{(2) h a d}$ using 
such fits are very stable as the fit range is varied, allowing far greater confidence in the reliability of the result. In particular we conclude that using a fit form (3.2) with the mass of the first pole fixed to the ground-state vector meson mass to be the optimal method of describing the lattice data for the hadronic vacuum polarisation.

In Fig. $1 \mathrm{~b}$ we see the value of the fit parameter $m_{1}$ from (3.2) as determined from fits to the lattice vacuum polarisation. The value of $m_{\mathrm{V}}$ obtained in [12] is shown in green, and this defines the band in which $m_{1}$ was constrained to reside in the fixed version of this fit.

\section{Results}

We extract our final results from the fit using (3.2) with the first mass fixed to that of the vector meson as measured on each ensemble. Observing the behaviour of the reduced $\chi^{2}$ as the fit range is varied, we choose a suitable value for $Q_{C}^{2}$ for each ensemble which provides the most reliable result. We attempt to choose a cut which provides a low reduced $\chi^{2}$ preferably where the parameter $m_{1}$ agrees without tension with $m_{\mathrm{V}}$.

These results are also shown as a function of $m_{\pi}^{2}$ in Fig. 2a, where we compare them to previous $2+1$ flavour results from [6]. Also shown is an extrapolation to the physical point, using a quadratic chiral ansatz. This produces a final result for the leading order hadronic vacuum polarisation contribution the anomalous magnetic moment of the muon

$$
a_{\mu}^{(2) h a d}=641(33) \times 10^{-10} .
$$

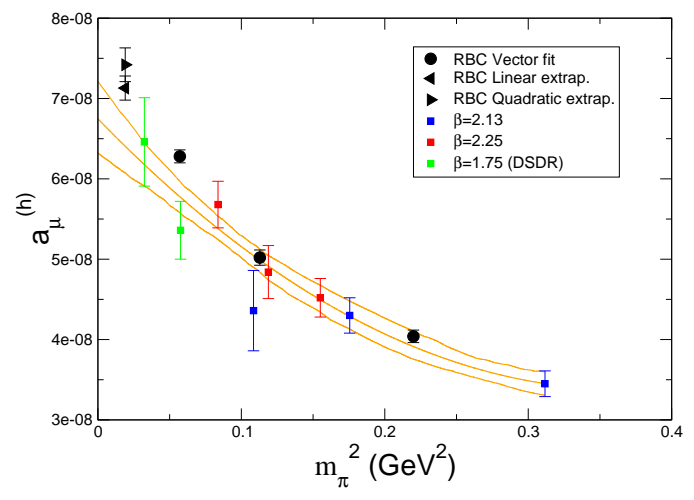

(a) Results using unmodified prescription, $H=1$.

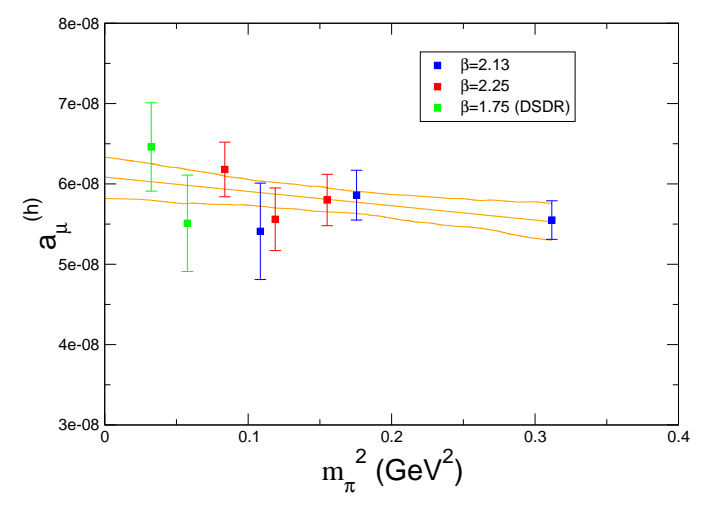

(b) Results using modified prescription $H=m_{V}$.

Figure 2: Integrated result for $a_{\mu}^{(2) h a d}$ as a function of the pseudoscalar mass squared.

We have also investigated the effect of modifying the kernel function in the integrand (1.1) in the manner outlined in [8], where in an effort to moderate the variation of the outcome of the integral as a function of the quark mass, the momentum argument of the kernel function is rescaled by a factor of the ratio of the value of a relevant observable $H$ (the mass of the vector meson appears to be an optimal choice) measured at the simulated quark mass to its physical value. This effectively defines the calculation of a new quantity which approaches the desired $a_{\mu}^{(2) h a d}$ in the 
physical limit. We show the results of such a calculation in Fig. 2b, along with an accompanying chiral extrapolation. The chiral variation in this redefined quantity is such that it allows for a linear extrapolation in quark mass. This method does indeed moderate the chiral behaviour of the result, however it has little effect on our data at light quark masses, primarily because the lattice vector meson masses are very near that of the physical $\rho$ meson, and, as of now, are not determined to any great precision on these lattices. As such this technique does not improve our chiral fit at this time, producing a compatible result with a similar uncertainty:

$$
a_{\mu}^{(2) h a d}=605(24) \times 10^{-10}
$$

\section{Conclusions}

We present a fully dynamical calculation of the leading-order hadronic vacuum polarisation contribution to the anomalous magnetic moment of the muon, using a 2+1 flavour simulation lattice QCD using domain wall fermions. Although we have an expensive fermion discretisation, we improve the accuracy of our result by convolving an accurate determination of the ground-state vector meson mass with our determination of the lattice hadronic vacuum polarisation in order to suppress the systematic uncertainty associated with the choice of fit ansatz. Our chiral extrapolation involves lattices at different bare couplings, and thus different lattice spacings, however at this level of precision we do not detect any significant discretisation, or finite volume errors in our result. Our final result we take to be

$$
a_{\mu}^{(2) h a d}=641(33)(32) \times 10^{-10}
$$

where the first error is statistical and the second is an estimate of the systematic error arising from the extrapolation to the chiral limit, taken as $5 \%$, motivated by the variation between the results (4.1) and (4.2). Our largest systematic uncertainty arises from the omission of the disconnected contributions and is of the order of $10 \%$ [15]. In order to obtain a more comprehensive and accurate result, we must include the disconnected contributions in our calculation. Furthermore, this being a first effort at deducing this quantity from our lattices, we have plans to improve it in a number of ways. In addition to the enhancement of our statistics, we would like to obtain a higher momentum resolution through the use of twisted boundary conditions, and also to explore the use of stochastic sources to further enhance our signal.

\section{Acknowledgements}

The calculations reported here were performed on the QCDOC computers [16, 17] at Columbia University, Edinburgh University, and at Brookhaven National Laboratory (BNL), Argonne Leadership Class Facility (ALCF) BlueGene/P resources at Argonne National Laboratory (ANL), and also the resources of the STFC-funded DiRAC facility. We wish to acknowledge support from STFC grant ST/H008845/1. At BNL, the QCDOC computers of the RIKEN-BNL Research Center and the USQCD Collaboration were used. The very large scale capability of the ALCF (supported by the Office of Science of the U.S. Department of Energy under contract DE-AC02-06CH11357) was critical for carrying out the challenging calculations reported here. The BAGEL library [18] was utilised extensively in these calculations. EK is supported by SUPA (The Scottish Universities 
Physics Alliance). JZ is supported by STFC grant ST/F009658/1. This work was supported in part by EU grant 238353 (STRONGnet). Data used at $\beta=1.75$ is to be presented in an upcoming publication [10], and we offer thanks to Chris Kelly for supplying preliminary results on these lattices.

\section{References}

[1] Peter Boyle, Luigi Del Debbio, Eoin Kerrane, and James Zanotti. Lattice Determination of the Hadronic Contribution to the Muon $g-2$ using Dynamical Domain Wall Fermions. 2011, 1107.1497. * Temporary entry*.

[2] F. Jegerlehner. Muon g - 2 update. Nucl. Phys. Proc. Suppl., 181-182:26-31, 2008.

[3] T. Blum. Lattice calculation of the lowest order hadronic contribution to the muon anomalous magnetic moment. ((U)). Phys. Rev. Lett., 91:052001, 2003, hep-lat/0212018.

[4] Göckeler, M. and others. Vacuum polarisation and hadronic contribution to muon g-2 from lattice QCD. Nucl. Phys., B688:135-164, 2004, hep-lat/0312032.

[5] T. Blum. Lattice calculation of the lowest order hadronic contribution to the muon anomalous magnetic moment: An update with Kogut-Susskind fermions. Nucl. Phys. Proc. Suppl., 129:904-906, 2004, hep-lat/0310064.

[6] C. Aubin and T. Blum. Calculating the hadronic vacuum polarization and leading hadronic contribution to the muon anomalous magnetic moment with improved staggered quarks. Phys. Rev., D75:114502, 2007, hep-lat/0608011.

[7] Michele Della Morte, Benjamin Jager, Andreas Juttner, and Hartmut Wittig. The leading hadronic vacuum polarisation on the lattice. AIP Conf.Proc., 1343:337-339, 2011, 1011.5793.

[8] Xu Feng, Karl Jansen, Marcus Petschlies, and Dru B. Renner. Two-flavor QCD correction to lepton magnetic moments at leading-order in the electromagnetic coupling. 2011, 1103.4818.

[9] Shigemi Ohta. Nucleon structure from $2+1$ flavor domain wall QCD at nearly physical pion mass. AIP Conf.Proc., 1354:148-154, 2011, 1102.0551.

[10] RBC/UKQCD. Continuum Limit Physics from 2+1 Flavor Domain Wall QCD II.

[11] C. Allton et al. Physical Results from 2+1 Flavor Domain Wall QCD and SU(2) Chiral Perturbation Theory. Phys. Rev., D78:114509, 2008, 0804.0473.

[12] Y. Aoki et al. Continuum Limit Physics from 2+1 Flavor Domain Wall QCD. 2010, 1011.0892.

[13] Vadim Furman and Yigal Shamir. Axial symmetries in lattice QCD with Kaplan fermions. Nucl. Phys., B439:54-78, 1995, hep-lat/9405004.

[14] Della Morte, Michele and Jüttner, Andreas. Quark disconnected diagrams in chiral perturbation theory. JHEP, 11:154, 2010, 1009.3783.

[15] Jüttner, Andreas and Della Morte, Michele. New ideas for g-2 on the lattice. PoS, LAT2009:143, 2009, 0910.3755 .

[16] P. Boyle et al. The QCDOC project. Nucl. Phys. Proc. Suppl., 140:169-175, 2005.

[17] P. A. Boyle, C. Jung, and T. Wettig. The QCDOC supercomputer: Hardware, software, and performance. ECONF, C0303241:THIT003, 2003, hep-lat/0306023.

[18] Peter A. and Boyle. The bagel assembler generation library. Computer Physics Communications, 180(12):2739 - 2748, 2009. 